J. Heittokangas, R. Korhonen and J. Rättyä

Nagoya Math. J.

Vol. 187 (2007), 91-113

\title{
LINEAR DIFFERENTIAL EQUATIONS WITH SOLUTIONS IN THE DIRICHLET TYPE SUBSPACE OF THE HARDY SPACE
}

\author{
J. HEITTOKANGAS, R. KORHONEN AND J. RÄTTŸ̈
}

\section{Dedicated to Nikolaos Danikas}

\begin{abstract}
Sufficient conditions for the analytic coefficients of the linear differential equation

$$
f^{(k)}+A_{k-1}(z) f^{(k-1)}+\cdots+A_{1}(z) f^{\prime}+A_{0}(z) f=0
$$

are found such that all solutions belong to a given $H_{q}^{\infty}$-space, or to the Dirichlet type subspace $\mathcal{D}^{p}$ of the classical Hardy space $H^{p}$, where $0<p \leq 2$. For $0<q<\infty$, the space $H_{q}^{\infty}$ consists of those functions $f$, analytic in the unit disc $D$, for which $|f(z)|\left(1-|z|^{2}\right)^{q}$ is uniformly bounded in $D$, and $f \in \mathcal{D}^{p}$ if the integral $\int_{D}\left|f^{\prime}(z)\right|^{p}\left(1-|z|^{2}\right)^{p-1} d \sigma_{z}$ converges.
\end{abstract}

\section{§1. Introduction}

The growth of entire solutions of the linear differential equation

$$
f^{(k)}+A_{k-1}(z) f^{(k-1)}+\cdots+A_{1}(z) f^{\prime}+A_{0}(z) f=0
$$

with entire coefficients is relatively well known in the complex plane [15], [20], [23], [32], where efficient tools, such as Wiman-Valiron and Nevanlinna theories, are available. As for local considerations, Nevanlinna theory has been applied to fast growing analytic solutions [3], [4], [7], [8], [16], [18], [21], [22], but the analysis of slowly growing solutions seems to require a different approach [16], [17], [19], [26], [30].

Chr. Pommerenke [26] studied the second-order equation

$$
f^{\prime \prime}+A(z) f=0,
$$

where $A(z)$ is an analytic function in the unit disc $D=\{z:|z|<1\}$.

Received August 9, 2005.

2000 Mathematics Subject Classification: Primary 34M10; Secondary 30D50, 30D55. 
Theorem A. ([26, Theorem 2]) Let $A(z)$ be an analytic function in $D$. There is an absolute constant $\alpha>0$ with the following property: If

$$
\sup _{a \in D} \int_{D}|A(z)|^{2}\left(1-|z|^{2}\right)^{3} \frac{1-|a|^{2}}{|1-\bar{a} z|^{2}} d \sigma_{z} \leq \alpha,
$$

then all solutions of (1.2) belong to the Hardy space $H^{2}$.

The definitions of the Hardy spaces and other relevant concepts are postponed to Section 2 below.

Theorem B. ([26, Theorem 3]) Let $A(z)$ be an analytic function in $D$, and let $0<\delta_{0}<1$. There is an absolute constant $\beta>0$ with the following property: If

$$
\sup _{0 \leq \theta \leq 2 \pi} \sup _{0<\delta \leq \delta_{0}} \frac{1}{\delta} \int_{1-\delta}^{1} \int_{\theta-\delta}^{\theta+\delta}\left|A\left(r e^{i t}\right)\right|^{2}(1-r)^{3} d t d r \leq \beta,
$$

then all solutions of (1.2) belong to the Hardy space $H^{2}$.

Pommerenke also pointed out that the constant $\alpha$ in Theorem A satisfies $\alpha \leq 9 \pi$. The following corollary offers a simpler but more restrictive condition on the coefficient $A(z)$ than (1.3) or (1.4).

Corollary C. ([26, Corollary 1]) Let $A(z)$ be an analytic function in $D$ satisfying $|A(z)| \leq \varphi(r)$ for $|z| \leq r<1$, where

$$
\int_{0}^{1} \varphi(r)^{2}(1-r)^{3} d r<\infty .
$$

Then all solutions of (1.2) belong to the Hardy space $H^{2}$.

Theorem 4 in [26] shows that, if the integral in (1.5) diverges, and if the function $\varphi(r)$ satisfies certain regularity conditions, then (1.2) possesses a solution $f$ which is not of bounded characteristic, and hence $f \notin H^{2}$.

The first author [16] studied the equation

$$
f^{(k)}+A(z) f=0,
$$

where $A(z)$ is analytic in $D$ and $k \in \mathbb{N}$. 
Theorem D. ([16, Theorem 4.3(2)]) If the analytic coefficient $A(z)$ of (1.6) satisfies $|A(z)|(1-|z|)^{k} \leq \alpha$ for all $z \in D$, then all solutions of (1.6) belong to $H_{q}^{\infty}$, where $q=\alpha /(k-1)$ !.

Theorem D essentially says that, for a given $q>0$, there exists an $\alpha$, depending only on $q$ and $k$, such that whenever $|A(z)|(1-|z|)^{k} \leq \alpha$, then all solutions of (1.6) belong to $H_{q}^{\infty}$.

The purpose of this study is to find sufficient conditions for the analytic coefficients of the linear differential equation (1.1) such that all solutions belong to a given weighted $H^{\infty}$-space, or to the Dirichlet type subspace $\mathcal{D}^{p}$ of the Hardy space $H^{p}$, where $0<p \leq 2$. In particular, the results obtained generalize Theorems A, B and D and Corollary C to equation (1.1). A number of related results are also presented including, for instance, boundary versions of the generalizations of Theorems A and D.

The remainder of the paper is organized as follows. The notation is fixed and the required function spaces are defined in Section 2. The results are presented and analyzed in Section 3, where some examples are also given. The necessary auxiliary results, which will be repeatedly used in the proofs of the results in Section 5, are listed in Section 4.

\section{§2. Notation}

Throughout the paper, $D(0, R)$ denotes the Euclidian disc of radius $R$ centered at the origin, so $D(0,1)=D$. For $0<p \leq \infty$, the Hardy space $H^{p}$ consists of those functions $f$, analytic in $D$, for which

$$
\|f\|_{H^{p}}=\sup _{0 \leq r<1} M_{p}(r, f)<\infty
$$

where

$$
M_{p}(r, f)=\left(\frac{1}{2 \pi} \int_{0}^{2 \pi}\left|f\left(r e^{i \theta}\right)\right|^{p} d \theta\right)^{1 / p}, \quad 0<p<\infty
$$

are the standard $L^{p}$-means of the restriction of $f$ to the circle of radius $r$ centered at the origin, and

$$
M_{\infty}(r, f)=M(r, f)=\max _{0 \leq \theta \leq 2 \pi}\left|f\left(r e^{i \theta}\right)\right| .
$$

Since $M_{p}(r, f)$ is an increasing function of $r$, the supremum in (2.1) is in fact the limit when $r$ tends to 1 . 
For $0<q<\infty$, the space $H_{q}^{\infty}$ (respectively $H_{q, 0}^{\infty}$ ) consists of those functions $f$, analytic in $D$, for which

$$
\|f\|_{H_{q}^{\infty}}=\sup _{0 \leq r<1} M(r, f)\left(1-r^{2}\right)^{q}<\infty
$$

(respectively $\left.\lim _{r \rightarrow 1^{-}}\left(1-r^{2}\right)^{q} M(r, f)=0\right)$.

The spaces $H_{q}^{\infty}$ (resp. $H_{q, 0}^{\infty}$ ) can be characterized in terms of the $\alpha$-Bloch spaces $\mathcal{B}^{\alpha}$ (resp. the little $\alpha$-Bloch spaces $\mathcal{B}_{0}^{\alpha}$ ) consisting of those functions $f$, analytic in $D$, for which

$$
\|f\|_{\mathcal{B}^{\alpha}}=\sup _{0 \leq r<1} M\left(r, f^{\prime}\right)\left(1-r^{2}\right)^{\alpha}<\infty, \quad 0<\alpha<\infty
$$

(resp. $\left.\lim _{r \rightarrow 1^{-}}\left(1-r^{2}\right)^{\alpha} M\left(r, f^{\prime}\right)=0,0<\alpha<\infty\right)$. Indeed, by Proposition $\mathrm{E}$ below, $H_{q}^{\infty}=\mathcal{B}^{q+1}$ and $H_{q, 0}^{\infty}=\mathcal{B}_{0}^{q+1}$ for all $0<q<\infty$. The classical Blochspace and the little Bloch-space are $\mathcal{B}=\mathcal{B}^{1}$ and $\mathcal{B}_{0}=\mathcal{B}_{0}^{1}$, respectively. The spaces $H^{p}, 1 \leq p<\infty$, and $\mathcal{B}^{\alpha}, 0<\alpha<\infty$, are Banach spaces with respect to the norms $\|f\|_{H^{p}}$ and $\|f\|_{\mathcal{B}^{\alpha}}+|f(0)|$, respectively.

Let the Green's function of $D$ with logarithmic singularity at $a \in D$ be denoted by $g(z, a)=-\log \left|\varphi_{a}(z)\right|$, where $\varphi_{a}(z)=(a-z) /(1-\bar{a} z)$ is the automorphism of $D$ which interchanges 0 and $a$. Straightforward calculations show that $\varphi_{a}$ is its own inverse and

$$
1-\left|\varphi_{a}(z)\right|^{2}=\left|\varphi_{a}^{\prime}(z)\right|\left(1-|z|^{2}\right)=\frac{\left(1-|a|^{2}\right)\left(1-|z|^{2}\right)}{|1-\bar{a} z|^{2}} .
$$

Moreover, let $d \sigma_{z}$ denote the element of the Lebesgue area measure on $D$.

For $0<p<\infty,-2<q<\infty$ and $0 \leq s<\infty$, the families $F(p, q, s)$ and $F_{0}(p, q, s)$ of function spaces consist of those functions $f$, analytic in $D$, for which

$$
\|f\|_{F(p, q, s)}=\left(\sup _{a \in D} \int_{D}\left|f^{\prime}(z)\right|^{p}\left(1-|z|^{2}\right)^{q} g^{s}(z, a) d \sigma_{z}\right)^{1 / p}<\infty
$$

and

$$
\lim _{|a| \rightarrow 1^{-}} \int_{D}\left|f^{\prime}(z)\right|^{p}\left(1-|z|^{2}\right)^{q} g^{s}(z, a) d \sigma_{z}=0, \quad 0<s<\infty,
$$

respectively. As usual, it is also defined $F_{0}(p, q, 0)=F(p, q, 0)$.

For $1 \leq p<\infty, F(p, q, s)$ is a Banach space with respect to the norm $\|f\|_{F(p, q, s)}+|f(0)|$, and so is $F_{0}(p, q, s)$ as a closed subspace of $F(p, q, s)$, see 
[33, Theorem 2.10 and Proposition 2.15]. The spaces $F(p, q, s)$, introduced by R. Zhao in [33], are known as the general family of function spaces. The importance of these spaces stems from the fact that, for appropriate parameter values $p, q$ and $s$, they coincide with several classical function spaces. For instance, it is well known that $F(2,1,0)=H^{2}$ and $F(p, p+q, 0)$ is the weighted Bergman space $A_{q}^{p}$, where $0<p<\infty$ and $-1<q<\infty$, consisting of those analytic functions $f$ in $D$ such that

$$
\|f\|_{A_{q}^{p}}=\left(\int_{D}|f(z)|^{p}\left(1-|z|^{2}\right)^{q} d \sigma_{z}\right)^{1 / p}<\infty
$$

see [11, Theorems 6 and 7] and [34, Theorem 4.2.9]. Moreover, $F(2,0, s)=$ $Q_{s}$ and $F_{0}(2,0, s)=Q_{s, 0}$, see [2], and, in particular, $F(2,0,1)=B M O A$ and $F_{0}(2,0,1)=V M O A$, the spaces of analytic functions with bounded and vanishing mean oscillation, respectively. For other relations, the reader is invited to see [28] and [33].

For $p>0$, the Dirichlet-type space $\mathcal{D}^{p}$ consists of those functions $f$, analytic in $D$, such that $f^{\prime} \in A_{p-1}^{p}$. Thus $\mathcal{D}^{p}=F(p, p-1,0)$ and, in particular, $\mathcal{D}^{2}=H^{2}$.

A positive measure $\mu$ on $D$ is a bounded $s$-Carleson measure, if

$$
\sup _{I} \frac{\mu(S(I))}{|I|^{s}}<\infty, \quad 0<s<\infty
$$

where $|I|$ denotes the arc length of a subarc $I$ of the boundary $\partial D$,

$$
S(I)=\{z \in D: z /|z| \in I, 1-|I| \leq|z|\}
$$

is the Carleson box based on $I$, and the supremum is taken over all subarcs $I$ of $\partial D$ such that $|I| \leq 1$. Moreover, if

$$
\lim _{|I| \rightarrow 0} \frac{\mu(S(I))}{|I|^{s}}=0, \quad 0<s<\infty,
$$

then $\mu$ is said to be a compact (vanishing) $s$-Carleson measure. When $s=1$, (2.3) and (2.4) reduce to the standard definitions of bounded and compact Carleson measures, respectively.

Two quantities $A$ and $B$ are comparable, if there exist a positive constant $C$ such that $C^{-1} B \leq A \leq C B$. 


\section{§3. Results}

Through the remainder of this paper the coefficients, and hence the solutions, of (1.1) are assumed to be analytic in $D$.

The first result generalizes Theorem $\mathrm{D}$ to the linear differential equation (1.1).

THEOREM 3.1. For every $q>0$ there exists a constant $\alpha=\alpha(q, k)>0$ such that if the coefficients $A_{j}(z)$ of (1.1) satisfy

$$
\left\|A_{j}\right\|_{H_{k-j}^{\infty}}=\sup _{z \in D}\left|A_{j}(z)\right|\left(1-|z|^{2}\right)^{k-j} \leq \alpha, \quad j=0, \ldots, k-1,
$$

then all solutions of (1.1) belong to $H_{q}^{\infty}$.

Three different proofs for Theorem 3.1 are given in Section 5.

Corollary 3.2. Let $0<p<\infty,-2<q<\infty$, and $0 \leq s \leq 1$ such that $(q+s+1) / p>1$. There exists a constant $\alpha=\alpha(p, q, s, k)>0$ such that if (3.1) holds, then all solutions of (1.1) belong to $F_{0}(p, q, s)$.

In the statement of Theorem 3.1 it is in fact sufficient to assume that the supremum in (3.1) is taken over an annulus $\{z \in D:|z| \geq \delta>0\}$.

TheOREM 3.3. Let $0<\delta<1$. For every $q>0$ there exists a constant $\alpha=\alpha(q, k)>0$ such that if the coefficients $A_{j}(z)$ of (1.1) satisfy

$$
\sup _{|z| \geq \delta}\left|A_{j}(z)\right|\left(1-|z|^{2}\right)^{k-j} \leq \alpha, \quad j=0, \ldots, k-1,
$$

then all solutions of (1.1) belong to $H_{q}^{\infty}$.

EXAMPLE 3.4. The functions

$$
f_{n}(z)=(1-z)^{\frac{1}{2}\left(-a_{1}+1+(-1)^{n} \sqrt{\left(a_{1}-1\right)^{2}+4 a_{0}}\right)}, \quad n=1,2,
$$

are linearly independent solutions of the differential equation

$$
f^{\prime \prime}-\frac{a_{1}}{1-z} f^{\prime}-\frac{a_{0}}{(1-z)^{2}} f=0,
$$

where $a_{0}, a_{1} \in \mathbb{R}$ such that $\left(a_{1}-1\right)^{2}+4 a_{0}>0$. If $a_{1}>q+1-a_{0} / q$ then $f_{1} \notin H_{q}^{\infty}$, and therefore the constant $\alpha$ in Theorems 3.1 and 3.3 satisfies

$$
\alpha \leq 2 \min _{a_{0} \in \mathbb{R}} \max \left\{\left|q+1-a_{0} / q\right|, 2\left|a_{0}\right|\right\}=\frac{4 q(q+1)}{2 q+1}
$$


in the case $k=2$. This clearly implies that $\alpha$ tends to zero as $q$ tends to zero.

The functions in $(3.3)$ and $f_{3}(z)=(1-z)^{2}$ are linearly independent solutions of

$$
f^{\prime \prime \prime}-\frac{a_{1}}{1-z} f^{\prime \prime}-\frac{a_{0}+a_{1}}{(1-z)^{2}} f^{\prime}-\frac{2 a_{0}}{(1-z)^{3}} f=0,
$$

where $a_{0}, a_{1} \in \mathbb{R}$. Therefore the constant $\alpha$ in Theorems 3.1 and 3.3 satisfies

$$
\begin{aligned}
\alpha & \leq 2 \min _{a_{0} \in \mathbb{R}} \max \left\{\left|q+1-a_{0} / q\right|, 2\left|q+1+a_{0}(1-1 / q)\right|, 8\left|a_{0}\right|\right\} \\
& = \begin{cases}\frac{16 q(q+1)}{3 q+1}, & 0<q \leq 1 \\
\frac{16 q(q+1)}{5 q-1}, & 1 \leq q<\infty\end{cases}
\end{aligned}
$$

in the case $k=3$. Once again this implies that $\alpha$ tends to zero as $q$ tends to zero.

As an immediate consequence of Theorem 3.3 it is deduced that if $\left|A_{j}(z)\right|\left(1-|z|^{2}\right)^{k-j}$ tends to zero as $z$ approaches the boundary $\partial D$, then all solutions of (1.1) belong to $H_{q}^{\infty}$ for all $q>0$.

Corollary 3.5. If $A_{j} \in H_{k-j, 0}^{\infty}$ for $j=0, \ldots, k-1$, then all solutions of (1.1) belong to $\bigcap_{0<q<\infty} H_{q}^{\infty}$.

ExAMPLE 3.6. The functions $f_{1}(z)=(1-z)^{1-\sqrt{2}}, f_{2}(z)=(1-z)^{1+\sqrt{2}}$ and $f_{3}(z)=1-z$ are linearly independent solutions of

$$
f^{\prime \prime \prime}+A_{1}(z) f^{\prime}+A_{0}(z) f=0
$$

where $A_{j}(z)=-(1-z)^{j-3}, j=0,1$. Clearly, $A_{j} \in H_{3-j}^{\infty} \backslash H_{3-j, 0}^{\infty}$ for $j=0,1$, yet $f_{1} \notin H_{q}^{\infty}$, if $q<\sqrt{2}-1$. This example shows the sharpness of Corollary 3.5 .

ExAmple 3.7. The functions $f_{1}(z)=1-z$ and $f_{2}(z)=-\log (1-z)$ are linearly independent solutions of

$$
f^{\prime \prime}+A_{1}(z) f^{\prime}+A_{0}(z) f=0
$$


where

$$
A_{j}(z)=\frac{1}{(\log (1-z)-1)(1-z)^{2-j}}, \quad j=0,1 .
$$

Clearly, $A_{j} \in H_{2-j, 0}^{\infty}$ for $j=0,1$, and $f_{1}, f_{2} \in \bigcap_{0<q<\infty} H_{q}^{\infty}$ as Corollary 3.5 claims.

Since the weighted Bergman space $A_{k-j-2}^{1}$ is included in $H_{k-j, 0}^{\infty}$, as seen by Lemma $\mathrm{G}$ below, the following consequence of Corollary 3.5 is obtained.

COROLlary 3.8. If the analytic coefficient $A_{j}(z)$ belongs to the weighted Bergman space $A_{k-j-2}^{1}$, that is, if

$$
\int_{D}\left|A_{j}(z)\right|\left(1-|z|^{2}\right)^{k-j-2} d \sigma_{z}<\infty, \quad j=0, \ldots, k-1,
$$

then all solutions of (1.1) belong to $\bigcap_{0<q<\infty} H_{q}^{\infty}$.

Two observations concerning Corollary 3.8 are made. First, condition (3.5) implies that the coefficient $A_{k-1}(z)$ must vanish identically. Second, if the exponent $k-j-2$ in condition (3.5) is increased to $k-j-1$, then all solutions of (1.1) belong to the Nevanlinna class by [19, Theorem 2.2], and this result is fairly sharp. Since the Nevanlinna class contains functions of exponential growth, unlike any $H_{q}^{\infty}$, Corollary 3.8 shows that in this case the situation changes radically when the exponent $k-j-1$ is decreased by one.

The following result is a generalization of Theorem A.

THEOREM 3.9. For every $0<p \leq 2$ there exists a constant $\alpha=$ $\alpha(p, k)>0$ such that if the coefficients $A_{j}(z)$ of (1.1) satisfy

$$
\sup _{a \in D} \int_{D}\left|A_{j}(z)\right|^{p}\left(1-|z|^{2}\right)^{p(k-j)-1} \frac{1-|a|^{2}}{|1-\bar{a} z|^{2}} d \sigma_{z} \leq \alpha, j=0, \ldots, k-1
$$

then all solutions of (1.1) belong to $\mathcal{D}^{p} \cap H_{p}^{\infty}$.

Since $\mathcal{D}^{2} \subset H_{2}^{\infty}$ and $\mathcal{D}^{2}=H^{2}$, Theorem 3.9 reduces to Theorem A for the second order equation (1.2). More generally, $\mathcal{D}^{p} \subset H_{1 / p}^{\infty} \subset H_{p}^{\infty}$ for $1 \leq p \leq 2$ by [33, Corollary 2.8] and Proposition E below. If, on the other hand, $0<p<1$, then neither of the spaces $H_{p}^{\infty}$ or $\mathcal{D}^{p}$ includes the other by [33, Propositions 5.8 and 5.9] and Proposition E. 
Note that Theorem 3.9 cannot be obtained by Corollary 3.2 since the condition $(q+s+1) / p>1$ is not satisfied $(q=p-1$ and $s=0)$, although the supremum in (3.1) is less than or equal to a constant times the supremum in (3.6), as one can see by applying Lemma $G$ together with the fact that the expressions $\left(1-|a|^{2}\right) /|1-\bar{a} z|^{2}$ and $1 /\left(1-|z|^{2}\right)$ are comparable when $z \in \Delta(a, r)$. In fact, the space $\mathcal{D}^{p}=F(p, p-1,0)$ is the largest among the spaces $F(p, q, s)$ such that $q+s+1 \leq p$. To see this, observe first that $F\left(p_{2}, q, s\right) \subset F\left(p_{1}, q, s\right)$ for $0<p_{1}<p_{2}<\infty$, and further, for $p=q+s+1$,

$$
\begin{aligned}
& \int_{D}\left|f^{\prime}(z)\right|^{p}\left(1-|z|^{2}\right)^{p-1} d \sigma_{z} \\
& \quad \leq \sup _{a \in D} \int_{D}\left|f^{\prime}(z)\right|^{p}\left(1-|z|^{2}\right)^{q}\left(1-\left|\varphi_{a}(z)\right|^{2}\right)^{s} d \sigma_{z} \\
& \quad \leq 2^{s} \sup _{a \in D} \int_{D}\left|f^{\prime}(z)\right|^{p}\left(1-|z|^{2}\right)^{q} g^{s}(z, a) d \sigma_{z}
\end{aligned}
$$

so that $F(p, q, s) \subset \mathcal{D}^{p}$ with $\|f\|_{\mathcal{D}^{p}} \leq 2^{s / p}\|f\|_{F(p, q, s)}$.

The following result says that it is sufficient that the conditions in (3.6) are satisfied for all $a$ close to the boundary $\partial D$.

TheOREM 3.10. Let $0<\delta<1$. For every $0<p \leq 2$ there exists a constant $\alpha=\alpha(p, k)>0$ such that if the coefficients $A_{j}(z)$ of (1.1) satisfy

$$
\sup _{|a| \geq \delta} \int_{D}\left|A_{j}(z)\right|^{p}\left(1-|z|^{2}\right)^{p(k-j)-1} \frac{1-|a|^{2}}{|1-\bar{a} z|^{2}} d \sigma_{z} \leq \alpha, j=0, \ldots, k-1,
$$

then all solutions of (1.1) belong to $\mathcal{D}^{p} \cap H_{p}^{\infty}$.

Observing carefully the proofs of Theorems 3.10 and 3.12, it follows that

$$
\|f\|_{\mathcal{D}^{p}} \leq C\left(\sum_{j=0}^{k-1} C\left(A_{j}\right)\right)\left(\|f\|_{H^{p}}+\sum_{j=1}^{k-1}\left|f^{(j)}(0)\right|\right),
$$

where $C>0$ depends only on $p$ and $k$, and

$$
C\left(A_{j}\right)=\sup _{a \in D} \int_{D}\left|A_{j}(z)\right|^{p}\left(1-|z|^{2}\right)^{p(k-j)-1} \frac{1-|a|^{2}}{|1-\bar{a} z|^{2}} d \sigma_{z} .
$$

If $2 \leq p<\infty$, inequality (3.8), with $C \sum C\left(A_{j}\right)$ being replaced by an absolute constant, holds for all analytic functions $f$ by a classical theorem 
of Littlewood and Paley [24]. However, if $0<p<2$, inequality (3.8) together with Lemma $\mathrm{J}$ below implies that $\|f\|_{H^{p}}$ and $\|f\|_{\mathcal{D}^{p}}$ must be finite or infinite at the same time. In other words, if the supremum in (3.6) is finite for all $A_{j}(z)$ (that is $A_{j} \in F(p, p(k-j+1)-2,1)$ by Theorem $\mathrm{F}$ below) then none of the solutions of (1.1) belongs to $H^{p} \backslash \mathcal{D}^{p}$.

Corollary 3.11. Let $0<p \leq 2$. If the coefficients $A_{j}(z)$ of $(1.1)$ satisfy

(3.9) $\lim _{|a| \rightarrow 1^{-}} \int_{D}\left|A_{j}(z)\right|^{p}\left(1-|z|^{2}\right)^{p(k-j)-1} \frac{1-|a|^{2}}{|1-\bar{a} z|^{2}} d \sigma_{z}=0, \quad j=0, \ldots, k-1$,

then all solutions of (1.1) belong to

$$
\mathcal{D}^{p} \cap\left(\bigcap_{0<q<\infty} H_{q}^{\infty}\right) .
$$

Note that condition (3.9) is equivalent to $A_{j} \in F_{0}(p, p(k-j+1)-2,1)$, $j=0, \ldots, k-1$, by [29, Theorem 3.3] and (2.2).

Theorems 3.12 and 3.13 below generalize Theorem B.

THEOREM 3.12. Let $0<\delta<1$. For every $0<p \leq 2$ there exists a constant $\beta=\beta(p, k)>0$ such that if the coefficients $A_{j}(z)$ of (1.1) satisfy

$$
\begin{aligned}
\sup _{0<|I| \leq \delta} \frac{1}{|I|} \int_{S(I)}\left|A_{j}^{(n)}(z)\right|^{p}\left(1-|z|^{2}\right)^{p(k+n-j)-1} d \sigma_{z} & \leq \beta \\
& j=0, \ldots, k-1
\end{aligned}
$$

for all $n=0, \ldots, k-1$, then all solutions of $(1.1)$ belong to $\mathcal{D}^{p} \cap H_{p}^{\infty}$.

In the special case (1.6) it is enough that (3.10) is satisfied only for $n=0$, as is seen by observing carefully the proof of Theorem 3.12.

ThEOREM 3.13. Let $0<\delta<1$. For every $0<p \leq 2$ there exists a constant $\beta=\beta(p, k)>0$ such that if the coefficient $A(z)$ of (1.6) satisfies

$$
\sup _{0<|I| \leq \delta} \frac{1}{|I|} \int_{S(I)}|A(z)|^{p}\left(1-|z|^{2}\right)^{p k-1} d \sigma_{z} \leq \beta,
$$

then all solutions of (1.6) belong to $\mathcal{D}^{p} \cap H_{p}^{\infty}$. 
The following immediate consequence of [29, Theorem 5.3] and Corollary 3.11 generalizes and improves Corollary C.

Corollary 3.14. Let $0<p \leq 2$. If the coefficients $A_{j}(z)$ of (1.1) satisfy

$$
\int_{0}^{1} M\left(r, A_{j}^{(n)}\right)^{p}\left(1-r^{2}\right)^{p(k-j+n)-1} d r<\infty, \quad j=0, \ldots, k-1,
$$

for some $n=n(j) \in \mathbb{N} \cup\{0\}$, then all solutions of (1.1) belong to

$$
\mathcal{D}^{p} \cap\left(\bigcap_{0<q<\infty} H_{q}^{\infty}\right) .
$$

Although in general (3.9) is weaker than (3.12), the conditions are equivalent if the Maclaurin series expansion of $A_{j}(z)$ has Hadamard gaps [29, Theorem 5.5].

Corollary 3.14 has the following consequence which is obtained by applying [11, Lemma 3].

Corollary 3.15. If the coefficients $A_{j}(z)$ of (1.1) satisfy

$$
\int_{0}^{1} M\left(r, A_{j}^{(n)}\right)^{1 /(k-j+n)} d r<\infty, \quad j=0, \ldots, k-1,
$$

for some $n=n(j) \in \mathbb{N} \cup\{0\}$, then all solutions of (1.1) belong to

$$
\left(\bigcap_{m \leq p \leq 2} \mathcal{D}^{p}\right) \cap\left(\bigcap_{0<q<\infty} H_{q}^{\infty}\right),
$$

where $m=\max _{j=0, \ldots, k-1}(k-j+n(j))^{-1}$.

The case $p=1$ in Corollary 3.14 can be further improved if the condition (3.12) is satisfied for $n=0$.

Corollary 3.16. If the coefficients $A_{j}(z)$ of (1.1) satisfy

$$
\int_{0}^{1} M\left(r, A_{j}\right)\left(1-r^{2}\right)^{k-j-1} d r<\infty, \quad j=0, \ldots, k-1,
$$

then all solutions of (1.1) belong to $\mathcal{D}^{1} \cap H^{\infty}$. 
Since clearly $\mathcal{D}^{1}$ contains unbounded functions and $H^{\infty}$ is not included in $\mathcal{D}^{1}$ by [27], neither of the spaces $\mathcal{D}^{1}$ and $H^{\infty}$ contains the other.

This section is concluded with a corollary which is an immediate consequence of Corollary 3.16 since (3.15) below implies (3.14) by [11, Lemma 3], see the proof of Corollary 3.15.

Corollary 3.17. If the coefficients $A_{j}(z)$ of (1.1) satisfy

$$
\int_{0}^{1} M\left(r, A_{j}\right)^{1 /(k-j)} d r<\infty, \quad j=0, \ldots, k-1,
$$

then all solutions of (1.1) belong to $\mathcal{D}^{1} \cap H^{\infty}$.

Corollary 3.17 can be proved in the following alternative way: An application of [17, Theorem 5.1] shows that the solutions of (1.1) are bounded. Moreover, the assumption (3.15) implies that the solutions belong to $\bigcap_{1 \leq p \leq 2} \mathcal{D}^{p} \subset \mathcal{D}^{1}$ by Corollary 3.15. However, since the solutions are bounded, $H^{\infty} \subset \mathcal{B}$ and $\mathcal{D}^{p_{1}} \cap \mathcal{B} \subset \mathcal{D}^{p_{2}} \cap \mathcal{B}$ with $\|f\|_{\mathcal{D}^{p_{2}}}^{p_{2}} \leq\|f\|_{\mathcal{B}}^{p_{2}-p_{1}}\|f\|_{\mathcal{D}^{p_{1}}}^{p_{1}}$ for $0<p_{1}<p_{2}<\infty$, it follows that

$$
\left(\bigcap_{1 \leq p \leq 2} \mathcal{D}^{p}\right) \cap H^{\infty}=\mathcal{D}^{1} \cap H^{\infty}
$$

\section{§4. Auxiliary results}

First, some $n$-th derivative characterizations of functions in $H_{q}^{\infty}$ and $F(p, q, s)$ are recalled.

Proposition E. ([35, Proposition 7]) Let $f$ be an analytic function in $D, 1<\alpha<\infty$ and $n \in \mathbb{N}$. Then the following quantities are comparable:

(1) $\|f\|_{H_{\alpha-1}^{\infty}}$,

(2) $\|f\|_{\mathcal{B}^{\alpha}}+|f(0)|$,

(3) $\sup _{z \in D}\left|f^{(n)}(z)\right|\left(1-|z|^{2}\right)^{n-1+\alpha}+\sum_{j=0}^{n-1}\left|f^{(j)}(0)\right|$.

TheOrem F. Let $f$ be an analytic function in $D$, and let $0<p<\infty$, $-2<q<\infty$ and $0 \leq s \leq 1$. Let $n \in \mathbb{N}$ and $q+s>-1$, or $n=0$ and $p<q+s+1$. Denote $d \mu(z)=\left|f^{(n)}(z)\right|^{p}\left(1-|z|^{2}\right)^{n p-p+q+s} d \sigma_{z}$. Then the following quantities are comparable: 
(1) $\|f\|_{F(p, q, s)}^{p}+|f(0)|^{p}$,

(2) $\sup _{a \in D} \int_{D}\left|f^{(n)}(z)\right|^{p}\left(1-|z|^{2}\right)^{n p-p+q} g^{s}(z, a) d \sigma_{z}+\sum_{j=0}^{n-1}\left|f^{(j)}(0)\right|^{p}$,

(3) $\sup _{a \in D} \int_{D}\left|f^{(n)}(z)\right|^{p}\left(1-|z|^{2}\right)^{n p-p+q}\left(1-\left|\varphi_{a}(z)\right|^{2}\right)^{s} d \sigma_{z}+\sum_{j=0}^{n-1}\left|f^{(j)}(0)\right|^{p}$,

(4) $\sup _{I} \frac{\mu(S(I))}{|I|^{s}}+\sum_{j=0}^{n-1}\left|f^{(j)}(0)\right|^{p}$,

and there are no terms in the sums if $n=0$.

For the proof of Theorem F, see [14], [28] and [29].

Theorem F, combined with the definition of $s$-Carleson measures, implies that $f \in F(p, q, s)$ if and only if the positive measure $\mu$ such that $d \mu(z)=\left|f^{(n)}(z)\right|^{p}\left(1-|z|^{2}\right)^{n p-p+q+s} d \sigma_{z}$ is a bounded $s$-Carleson measure.

The following lemma is obtained by a straightforward calculation, see, for instance, [33, p. 18].

LEMmA G. Let $f$ be an analytic function in $D$, and let $0<p<\infty$, $0<q<\infty, 0<r<1$ and $a \in D$. Then there is a constant $C>0$, depending only on $q$ and $r$, such that

$$
|f(a)|^{p}\left(1-|a|^{2}\right)^{q} \leq C \int_{\Delta(a, r)}|f(z)|^{p}\left(1-|z|^{2}\right)^{q-2} d \sigma_{z}
$$

where $\Delta(a, r)=\left\{z \in D:\left|\varphi_{a}(z)\right|<r\right\}$.

The following annulus version of Carleson's theorem [5], [6], [9], [10], $[12]$ is needed.

Theorem H. Let $\mu$ be a positive measure on D, and let $0<p<\infty$ and $0 \leq r<1$. Then $\mu$ is a bounded Carleson measure if and only if there is a constant $C>0$, depending only on $p$, such that

$$
\int_{D \backslash D(0, r)}|f(z)|^{p} d \mu(z) \leq C\|f\|_{H^{p}}^{p}
$$

for all analytic functions $f$ in $D$, in particular for all $f \in H^{p}$. Moreover, if $\mu$ is a bounded Carleson measure, then $C=C_{1} C_{2}$, where $C_{1}>0$ is an absolute constant and

$$
C_{2}=\sup _{|I| \leq 1-r} \frac{\mu(S(I))}{|I|}
$$


If $r=0$, then Theorem $\mathrm{H}$ reduces to Carleson's theorem. If $0<r<1$, then an application of Carleson's theorem to the measure $\mu_{r}$ such that $d \mu_{r}(z)=\chi_{D \backslash D(0, r)} d \mu(z)$ shows that $C$ is of the form $C=C_{1} C_{2}$, where $\chi_{E}$ is the characteristic function of the set $E, C_{1}>0$ is an absolute constant, and

$$
C_{2}=\sup _{I} \frac{\mu(S(I) \backslash D(0, r))}{|I|} .
$$

Then the following lemma yields (4.2).

Lemma 4.1. Let $\mu$ be a positive measure on $D$, and let $r \in(0,1)$. Then

$$
\sup _{I} \frac{\mu(S(I) \backslash \Delta(0, r))}{|I|} \leq 2 \sup _{|I| \leq 1-r} \frac{\mu(S(I))}{|I|} .
$$

Proof. Let $I=\left\{e^{i \phi}: \theta \leq \phi \leq \theta+|I|\right\}$ be a subarc of $\partial D$ such that $|I| \leq 1$. If $|I| \leq 1-r$, then clearly

$$
\frac{\mu(S(I) \backslash \Delta(0, r))}{|I|}=\frac{\mu(S(I))}{|I|} \leq \sup _{|I| \leq 1-r} \frac{\mu(S(I))}{|I|} .
$$

If $|I|>1-r$, let $n=\max \{k \in \mathbb{N}: k(1-r)<|I|\}$, and define $I_{j}=\left\{e^{i \phi}:\right.$ $\theta+j(1-r) \leq \phi \leq \theta+(j+1)(1-r)\}$ for $j=0, \ldots, n$. Then $\left|I_{j}\right|=1-r$ for $j=0, \ldots, n$, and therefore

$$
\begin{aligned}
& \frac{\mu(S(I) \backslash \Delta(0, r))}{|I|}=\frac{1}{|I|} \int_{S(I)} \chi_{D \backslash \Delta(0, r)}(z) d \mu(z) \\
& \leq \frac{1}{|I|}\left(\sum_{j=0}^{n-1} \int_{S\left(I_{j}\right)} d \mu(z)+\int_{S\left(I_{n}\right)} \chi_{D \backslash \Delta(0, r)}(z) d \mu(z)\right) \\
& \leq \frac{1}{n}\left(\sum_{j=0}^{n} \frac{1}{\left|I_{j}\right|} \int_{S\left(I_{j}\right)} d \mu(z)\right) \\
& \leq \frac{n+1}{n} \sup _{|I| \leq 1-r} \frac{\mu(S(I))}{|I|} \leq 2 \sup _{|I| \leq 1-r} \frac{\mu(S(I))}{|I|} .
\end{aligned}
$$

The assertion follows by (4.4) and (4.5).

The next two lemmas contain some basic properties of Carleson measures. 
Lemma I. ([1, Lemma 2.1]) Let $\mu$ be a positive measure on D, and let $0<s<\infty$. Then $\mu$ is a bounded $s$-Carleson measure if and only if

$$
\sup _{a \in D} \int_{D}\left|\varphi_{a}^{\prime}(z)\right|^{s} d \mu(z)<\infty
$$

Further, the supremum in (4.6) is comparable to the supremum in (2.3). Furthermore, $\mu$ is a compact $s$-Carleson measure if and only if

$$
\lim _{|a| \rightarrow 1^{-}} \int_{D}\left|\varphi_{a}^{\prime}(z)\right|^{s} d \mu(z)=0 .
$$

Lemma 4.2. Let $\mu$ be a positive measure on $D$, and let $t \in(0,1)$ and $0<s<\infty$. Then

$$
\sup _{|I| \leq 1-t} \frac{\mu(S(I))}{|I|^{s}} \leq 10^{s} \sup _{|a| \geq t} \int_{D}\left|\varphi_{a}^{\prime}(z)\right|^{s} d \mu(z) .
$$

Proof. Let $z \in S(I)$. Choose $a=(1-|I|) e^{i \theta}$, where $e^{i \theta}$ is the midpoint of $I$, so that every $I$ on $\partial D$ with $|I| \leq 1-t$ corresponds to a unique $a \in D$ with $|a| \geq t$, and vice versa. Since $|1-\bar{a} z| \leq 1-|a|^{2}+|z-a|$, $1-|a|^{2}=|I|(2-|I|)$, and

$$
\begin{aligned}
|z-a|^{2} & \leq\left|e^{i\left(\theta+\frac{|I|}{2}\right)}-(1-|I|) e^{i \theta}\right|^{2} \\
& =1-2(1-|I|) \operatorname{Re}\left(e^{-i \frac{|I|}{2}}\right)+(1-|I|)^{2} \\
& \leq 1-2(1-|I|)\left(1-\frac{|I|^{2}}{8}\right)+(1-|I|)^{2} \leq \frac{5}{4}|I|^{2},
\end{aligned}
$$

it follows that $\left|\varphi_{a}^{\prime}(z)\right| \geq(10|I|)^{-1}$ for all $z \in S(I)$. This yields inequality (4.7).

The following lemma gives a relation between $H^{p}$ - and $\mathcal{D}^{p}$-norms when $0<p \leq 2$.

Lemma J. ([31, Lemma 1.4], [11, Theorem $\left.\left.3^{\prime}\right]\right)$ Let $f$ be an analytic function in $D$, and let $0<p \leq 2$. Then there is a constant $C>0$, depending only on $p$, such that

$$
\|f\|_{H^{p}} \leq C\left(\|f\|_{\mathcal{D}^{p}}+|f(0)|\right) .
$$


If $p \geq 2$, then $\|f\|_{\mathcal{D}^{p}} \leq C\|f\|_{H^{p}}$ holds by a classical result of Littlewood and Paley [24], [25].

The following basic lemma is also needed in Section 5.

Lemma K. $([10$, p. 57$])$ Let $a_{n} \geq 0$ for $n=1, \ldots, N$. Then

$$
\left(\sum_{n=1}^{N} a_{n}\right)^{p} \leq\left(\sum_{n=1}^{N} a_{n}^{p}\right), \quad 0<p \leq 1,
$$

and

$$
\left(\sum_{n=1}^{N} a_{n}\right)^{p} \leq N^{p-1}\left(\sum_{n=1}^{N} a_{n}^{p}\right), \quad 1 \leq p<\infty .
$$

\section{$\S 5$. Proofs}

Proof of Theorem 3.1. Denote $f_{\rho}(z)=f(\rho z)$ and $A_{j, \rho}(z)=A_{j}(\rho z)$, where $1 / 2 \leq \rho<1$. Then Proposition E and the Leibnitz' formula with (1.1), see [17, eq. (3.6)], yields

$$
\begin{aligned}
\left\|f_{\rho}\right\|_{H_{q}^{\infty}} & \leq C_{1}\left(\left\|f_{\rho}^{(k)}\right\|_{H_{q+k}^{\infty}}+\sum_{j=0}^{k-1}\left|f^{(j)}(0)\right|\right) \\
& \leq C_{2}\left(\sum_{j=0}^{k-1} \sum_{i=0}^{j}\left\|\left(A_{j, \rho}^{(i)} f_{\rho}\right)^{(j-i)}\right\|_{H_{q+k}^{\infty}}+\sum_{j=0}^{k-1}\left|f^{(j)}(0)\right|\right) .
\end{aligned}
$$

By Proposition E again,

$$
\begin{aligned}
\sum_{j=0}^{k-1} \sum_{i=0}^{j}\left\|\left(A_{j, \rho}^{(i)} f_{\rho}\right)^{(j-i)}\right\|_{H_{q+k}^{\infty}} & \leq C_{3} \sum_{j=0}^{k-1} \sum_{i=0}^{j}\left\|A_{j, \rho}^{(i)} f_{\rho}\right\|_{H_{q+k-j+i}^{\infty}} \\
& \leq C_{3} \sum_{j=0}^{k-1} \sum_{i=0}^{j}\left\|A_{j, \rho}^{(i)}\right\|_{H_{k-j+i}^{\infty}}\left\|f_{\rho}\right\|_{H_{q}^{\infty}} \\
& \leq C_{4}\left\|f_{\rho}\right\|_{H_{q}^{\infty}} \sum_{j=0}^{k-1} j\left\|A_{j, \rho}\right\|_{H_{k-j}^{\infty}} \\
& \leq C_{5} \alpha\left\|f_{\rho}\right\|_{H_{q}^{\infty}}
\end{aligned}
$$

It follows that

$$
\left(1-C_{6} \alpha\right)\left\|f_{\rho}\right\|_{H_{q}^{\infty}} \leq C_{6} \sum_{j=0}^{k-1}\left|f^{(j)}(0)\right|,
$$


where the constant $C_{6}$ depends only on $q$ and $k$. The assertion is obtained by choosing $\alpha$ sufficiently small and letting $\rho \rightarrow 1^{-}$.

Two alternative ways to prove Theorem 3.1 are given. An application of the growth estimate [17, Theorem 4.1(a)] and Proposition E together with the assumption (3.1) yields

$$
\left|f\left(r e^{i \theta}\right)\right| \leq \frac{C_{1}}{(1-r)^{\alpha C_{2}}}
$$

where $C_{1}$ and $C_{2}$ are positive constants such that $C_{1}$ depends on the initial values of $f$ at the origin, and $C_{2}$ depends on $k$ only. The assertion of Theorem 3.1 follows by (5.1). Theorem 3.1 can also be proved by applying [17, Theorem 5.1]. This approach does not require the use of Proposition E, and it is chosen while proving the boundary version Theorem 3.3 below.

Proof of Corollary 3.2. Let $q_{0}>0$ be small enough so that $1+q_{0}<$ $(q+s+1) / p$. By Theorem 3.1, there exists a constant $\alpha>0$ such that if (3.1) holds, then all solutions of (1.1) belong to $H_{q_{0}}^{\infty}$. Now, by Proposition E and [33, Proposition 5.7], it follows that $H_{q_{0}}^{\infty}=\mathcal{B}^{q_{0}+1} \subset F_{0}(p, q, s)$, which yields the assertion.

Proof of Theorem 3.3. Without loss of generality, assume that $\alpha \leq 1$ and $\delta<r<1$. By [17, Theorem 5.1] there is a constant $C_{1}>0$, depending only on the initial values of $f$, such that

$$
\left|f\left(r e^{i \theta}\right)\right| \leq C_{1} \exp \left(k \int_{0}^{r} \sum_{j=0}^{k-1}\left|A_{j}\left(s e^{i \theta}\right)\right|^{1 /(k-j)} d s\right)
$$

for all $\theta \in[0,2 \pi]$, and so the assumption (3.2) yields

$$
\begin{aligned}
\left|f\left(r e^{i \theta}\right)\right| \leq C_{1} \exp \left(k \int_{0}^{\delta} \sum_{j=0}^{k-1}\left|A_{j}\left(s e^{i \theta}\right)\right|^{1 /(k-j)} d s\right. \\
\left.\quad+k \int_{\delta}^{r} \sum_{j=0}^{k-1}\left|A_{j}\left(s e^{i \theta}\right)\right|^{1 /(k-j)} d s\right) \\
=C_{2} \exp \left(k \int_{\delta}^{r} \sum_{j=0}^{k-1}\left|A_{j}\left(s e^{i \theta}\right)\right|^{1 /(k-j)} d s\right) \\
\leq C_{2} \exp \left(\alpha^{1 / k} k^{2} \int_{\delta}^{r} \frac{d s}{1-s}\right) \leq C_{2} \frac{1}{(1-r)^{\alpha^{1 / k} k^{2}}}
\end{aligned}
$$


from which the assertion follows by choosing $\alpha=\left(q / k^{2}\right)^{k}$.

Proof of Theorem 3.9. The assertion follows by Theorem 3.10.

Proof of Theorem 3.10. By Lemma 4.2 and the reasoning in [28, pp. 4243] it follows that, for all $n=0, \ldots, k-1$ and $j=0, \ldots, k-1$,

$$
\begin{aligned}
& \sup _{|I| \leq 1-\delta} \frac{1}{|I|} \int_{S(I)}\left|A_{j}^{(n)}(z)\right|^{p}\left(1-|z|^{2}\right)^{p(k+n-j)-1} d \sigma_{z} \\
& \quad \leq 10 \sup _{|a| \geq \delta} \int_{D}\left|\varphi_{a}^{\prime}(z)\right|\left|A_{j}^{(n)}(z)\right|^{p}\left(1-|z|^{2}\right)^{p(k+n-j)-1} d \sigma_{z} \\
& \quad \leq C \sup _{|a| \geq \delta} \int_{D}\left|A_{j}(z)\right|^{p}\left(1-|z|^{2}\right)^{p(k-j)-1} \frac{1-|a|^{2}}{|1-\bar{a} z|^{2}} d \sigma_{z},
\end{aligned}
$$

where the constant $C>0$ depends only on $p$ and $k$. Hence, for $\alpha$ small enough, all solutions belong to $\mathcal{D}^{p} \cap H_{p}^{\infty}$ by Theorem 3.12.

Proof of Corollary 3.11. Theorem 3.10 implies that the solutions of (1.1) belong to $D^{p}$. The assumption (3.9) together with [33, Lemma 2.9] show that $A_{j} \in H_{k-j, 0}^{\infty}$ for $j=0, \ldots, k-1$. Therefore, by Corollary 3.5, all solutions of (1.1) belong to $\bigcap_{0<q<\infty} H_{q}^{\infty}$ also.

Proof of Theorem 3.12. By Lemma G and the fact that $1-|a|$ and $1-|z|$ are comparable for all $z \in \Delta(a, 1 / 2)$, it follows that, for all $j=0, \ldots, k-1$,

$$
\begin{aligned}
& \left|A_{j}(a)\right|^{p}\left(1-|a|^{2}\right)^{p(k-j)} \\
& \quad \leq \frac{C_{1}}{1-|a|} \int_{\Delta(a, 1 / 2)}\left|A_{j}(z)\right|^{p}\left(1-|z|^{2}\right)^{p(k-j)-1} d \sigma_{z},
\end{aligned}
$$

where $C_{1}$ depends only on $k$ and $p$. Let $I_{a}$ be the $\operatorname{arc}$ on the boundary of $D$ centered at $e^{i \arg a}$ with the length $\frac{3}{2}(1-|a|) /\left(1-\frac{|a|}{2}\right)$. Then $1-|a| \geq \frac{1}{3}\left|I_{a}\right|$, and since $\Delta(a, r), 0<r<1$, is a Euclidean disc centered at $\left(1-r^{2}\right) a /(1-$ $\left.|a|^{2} r^{2}\right)$ and of radius $\left(1-|a|^{2}\right) r /\left(1-|a|^{2} r^{2}\right)[12$, p. 3], the disc $\Delta(a, 1 / 2)$ is a subset of $S\left(I_{a}\right)$. Hence by (5.3),

$$
\begin{aligned}
& \sup _{|a| \geq \frac{3-2 \delta}{3-\delta}}\left|A_{j}(a)\right|^{p}\left(1-|a|^{2}\right)^{p(k-j)} \\
& \leq \sup _{|a| \geq \frac{3-2 \delta}{3-\delta}} \frac{C_{2}}{\left|I_{a}\right|} \int_{S\left(I_{a}\right)}\left|A_{j}(z)\right|^{p}\left(1-|z|^{2}\right)^{p(k-j)-1} d \sigma_{z} \\
& \quad=\sup _{|I| \leq \delta} \frac{C_{2}}{|I|} \int_{S(I)}\left|A_{j}(z)\right|^{p}\left(1-|z|^{2}\right)^{p(k-j)-1} d \sigma_{z},
\end{aligned}
$$


and it follows that, for $\alpha$ small enough in (3.10), all solutions of (1.1) belong to $H_{p}^{\infty}$ by Theorem 3.3 .

To show that all solutions belong to $\mathcal{D}^{p}$, let $1 / 2 \leq \rho<1, r=1-\delta$, and let $f$ be a solution of (1.1). Then, by Theorem $\mathrm{F}$ and the Leibnitz' formula,

$$
\begin{aligned}
& \left\|f_{\rho}\right\|_{\mathcal{D}^{p}}^{p} \leq C_{1}\left(\int_{D}\left|f^{(k)}(\rho z)\right|^{p}\left(1-|z|^{2}\right)^{p k-1} d \sigma_{z}+\sum_{j=1}^{k-1}\left|f^{(j)}(0)\right|^{p}\right) \\
& \leq C_{2}\left(\sum_{j=0}^{k-1} \sum_{n=0}^{j} \int_{D}\left|\left(A_{j}^{(n)}(\rho z) f(\rho z)\right)^{(j-n)}\right|^{p}\left(1-|z|^{2}\right)^{p k-1} d \sigma_{z}\right. \\
& \left.+\sum_{j=1}^{k-1}\left|f^{(j)}(0)\right|^{p}\right) \\
& \leq C_{3} \sum_{j=0}^{k-1} \sum_{n=0}^{j} \int_{D \backslash D(0, r)}|f(\rho z)|^{p}\left|A_{j}^{(n)}(\rho z)\right|^{p}\left(1-|z|^{2}\right)^{p(k+n-j)-1} d \sigma_{z}+C_{4},
\end{aligned}
$$

where

$$
\begin{gathered}
C_{4}=C_{3}\left(\sum_{j=0}^{k-1} \sum_{n=0}^{j} \int_{D(0, r)}|f(z)|^{p}\left|A_{j}^{(n)}(z)\right|^{p}\left(1-|z|^{2}\right)^{p(k+n-j)-1} d \sigma_{z}\right. \\
\left.+\sum_{j=1}^{k-1}\left|f^{(j)}(0)\right|^{p}\right)
\end{gathered}
$$

and the constant $C_{3}$ depends only on $p$ and $k$. Since by the assumption and Theorem $\mathrm{F}$ the positive measure $d \mu_{n, j, \rho}(z)=\left|A_{j}^{(n)}(\rho z)\right|^{p}(1-$ $\left.|z|^{2}\right)^{p(k+n-j)-1} d \sigma_{z}$ is a bounded Carleson measure for all $1 / 2 \leq \rho \leq 1$, $j=0, \ldots, k-1$ and $n=0, \ldots, k-1$, an application of Theorem $\mathrm{H}$ and Lemma J yields

$$
\begin{aligned}
\int_{D \backslash D(0, r)}|f(\rho z)|^{p} d \mu_{n, j, \rho}(z) & \leq C_{5} C_{6}(n, j, \rho)\left\|f_{\rho}\right\|_{H^{p}}^{p} \\
& \leq C_{6}(n, j, \rho) C_{7}\left(\left\|f_{\rho}\right\|_{\mathcal{D}^{p}}^{p}+|f(0)|^{p}\right),
\end{aligned}
$$

where $C_{5}$ and $C_{7}$ depend only on $p$, and

$$
\begin{aligned}
C_{6}(n, j, \rho) & =\sup _{|I| \leq 1-r} \frac{1}{|I|} \int_{S(I)} d \mu_{n, j, \rho}(z) \\
& =\sup _{|I| \leq \delta} \frac{1}{|I|} \int_{S(I)}\left|A_{j}^{(n)}(\rho z)\right|^{p}\left(1-|z|^{2}\right)^{p(k+n-j)-1} d \sigma_{z}
\end{aligned}
$$


Therefore, by (5.4), (5.5) and (5.6), and letting $\rho \rightarrow 1^{-}$, the assumption (3.10) yields

$$
\|f\|_{\mathcal{D}^{p}}^{p}\left(1-C_{8} \beta\right) \leq C_{8}|f(0)|^{p} \beta+C_{4},
$$

where $C_{8}$ depends only on $p$ and $k$. The assertion follows.

Proof of Corollary 3.15. By [11, Lemma 3] there is a constant $C>0$, depending only on $p, k, j$ and $n(j)$, such that

$$
\begin{aligned}
& \int_{0}^{1} M\left(r, A_{j}^{(n)}\right)^{p}\left(1-r^{2}\right)^{p(k-j+n)-1} d r \\
& \quad \leq C\left(\int_{0}^{1} M\left(r, A_{j}^{(n)}\right)^{1 /(k-j+n)} d r\right)^{p(k-j+n)}
\end{aligned}
$$

for all $p \geq 1 /(k-j+n(j))$, and the assertion follows by Corollary 3.14.

Proof of Corollary 3.16. By Corollary 3.14, all solutions belong to $\mathcal{D}^{1}$. Further, if $g$ is an analytic function in $D$, then by the Cauchy integral formula,

$$
\left|g^{\prime}\left(r e^{i \theta}\right)\right| \leq \frac{1}{2 \pi} \int_{0}^{2 \pi} \frac{\left|g\left(\rho e^{i(t+\theta)}\right)\right|}{\left|\rho e^{i t}-r\right|^{2}} d t,
$$

where $\rho=(1+r) / 2$, and hence

$$
\begin{array}{rl}
\int_{0}^{1} & M\left(r, g^{\prime}\right)(1-r)^{q} d r \\
\quad \leq & \int_{0}^{1}\left(\sup _{0 \leq \theta<2 \pi} \frac{1}{2 \pi} \int_{0}^{2 \pi} \frac{\left|g\left(\rho e^{i(t+\theta)}\right)\right|}{\left|\rho e^{i t}-r\right|^{2}} d t\right)(1-r)^{q} d r \\
\leq & \int_{0}^{1} M(\rho, g) \frac{(1-r)^{q}}{\rho^{2}-r^{2}} d r \\
\leq & 2^{q+2} \int_{0}^{1} M(\rho, g)(1-\rho)^{q-1} d \rho .
\end{array}
$$

Thus, by the assumption (3.14),

$$
\int_{0}^{1} M\left(r, A_{j}^{(n)}\right)\left(1-r^{2}\right)^{k-j+n-1} d r<\infty
$$

for all $j=0, \ldots, k-1$ and for all $n=0, \ldots, j$, and hence the fact that the solutions are bounded follows by [17, Theorem 4.1]. 
Acknowledgement. The authors wish to acknowledge partial support by the EU Research Training Network Contract No. HPRN-CT-200000116, the Academy of Finnish 204819 and 210245, MEC-Spain MTM200507347, Spanish Thematic Network MTM2006-26627-E, and the Väisälä Fund of the Finnish Academy of Science and Letters.

\section{REFERENCES}

[1] R. Aulaskari, D. Stegenga and J. Xiao, Some subclasses of BMOA and their characterization in terms of Carleson measures, Rocky Mountain J. Math., 26 (1996), 485-506.

[2] R. Aulaskari, J. Xiao and R. Zhao, On subspaces and subsets of BMOA and UBC, Analysis, 15 (1995), no. 2, 101-121.

[3] D. Benbourenane and L. Sons, On global solutions of complex differential equations in the unit disk, Complex Var. Theory Appl., 49 (2004), no. 13, 913-925.

[4] T.-B. Cao and H.-X. Yi, The growth of solutions of linear differential equations with coefficients of iterated order in the unit disc, J. Math. Anal. Appl., 319 (2006), no. 1, 278-294.

[5] L. Carleson, An interpolation problem for bounded analytic functions, Amer. J. Math., 80 (1958), 921-930.

[6] L. Carleson, Interpolations by bounded analytic functions and the corona problem, Ann. of Math., 76 (1962), no. 3, 547-559.

[7] Z.-X. Chen and K. H. Shon, The growth of solutions of differential equations with coefficients of small growth in the disc, J. Math. Anal. Appl., 297 (2004), no. 1, 285-304.

[8] I. Chyzhykov, G. G. Gundersen and J. Heittokangas, Linear differential equations and logarithmic derivative estimates, Proc. London Math. Soc., 86 (2003), no. 3, $735-754$.

[9] C. C. Cowen and B. D. MacCluer, Composition operators on spaces of analytic functions, Studies in Advanced Mathematics, CRC Press, Boca Raton, FL, 1995.

[10] P. Duren, Theory of $H^{p}$ Spaces, Academic Press, New York-London, 1970.

[11] T. M. Flett, The dual of an inequality of Hardy and Littlewood and some related inequalitites, J. Math. Anal. Appl., 38 (1972), 746-765.

[12] J. B. Garnett, Bounded analytic functions, Pure and Applied Mathematics, vol. 96, Academic Press, Inc., New York-London, 1981.

[13] D. Girela, Growth of the derivative of bounded analytic functions, Complex Variables, 20 (1992), no. 1-4, 221-227.

[14] F. Pérez-González and J. Rättyä, Forelli-Rudin estimates, Carleson measures and $F(p, q, s)$-functions, J. Math. Anal. Appl., 315 (2006), no. 2, 394-414.

[15] G. G. Gundersen, E. M. Steinbart and S. Wang, The possible orders of solutions of linear differential equations with polynomial coefficients, Trans. Amer. Math. Soc., 350 (1998), no. 3, 1225-1247. 
[16] J. Heittokangas, On complex differential equations in the unit disc, Ann. Acad. Sci. Fenn. Math. Diss., 122 (2000), 1-54.

[17] J. Heittokangas, R. Korhonen and J. Rättyä, Growth estimates for solutions of linear complex differential equations, Ann. Acad. Sci. Fenn., 29 (2004), 233-246.

[18] J. Heittokangas, R. Korhonen and J. Rättyä, Fast growing solutions of linear differential equations in the unit disc, Results Math., 49 (2006), 265-278.

[19] J. Heittokangas, R. Korhonen and J. Rättyä, Linear differential equations with coefficients in weighted Bergman and Hardy spaces, to appear in Trans. Amer. Math. Soc.

[20] E. Hille, Ordinary Differential Equations in the Complex Domain, Pure and Applied Mathematics, Wiley-Interscience (John Wiley \& Sons), New York-London-Sydney, 1976.

[21] R. Korhonen and J. Rättyä, Linear differential equations in the unit disc with analytic solutions of finite order, Proc. Amer. Math. Soc., 135 (2007), no. 5, 1355-1363.

[22] R. Korhonen and J. Rättyä, Finite order solutions of linear differential equations in the unit disc, preprint.

[23] I. Laine, Nevanlinna Theory and Complex Differential Equations, Walter de Gruyter, Berlin, 1993.

[24] J. E. Littlewood and R. E. A. C. Paley, Theorems on Fourier series and power series (II), Proc. London Math. Soc., 42 (1936), 52-89.

[25] D. Luecking, A new proof of an inequality of Littlewood and Paley, Proc. Amer. Math. Soc., 103 (1988), no. 3, 887-893.

[26] Chr. Pommerenke, On the mean growth of the solutions of complex linear differential equations in the disk, Complex Variables, 1 (1982), no. 1, 23-38.

[27] W. Rudin, The radial variation of analytic functions, Duke Math. J., 22 (1955), 235-242.

[28] J. Rättyä, On some complex function spaces and classes, Ann. Acad. Sci. Fenn. Math. Diss., 124 (2001), 1-73.

[29] J. Rättyä, $n$-th derivative characterizations, mean growth of derivatives and $F(p, q, s)$, Bull. Australian Math. Soc., 68 (2003), 405-421.

[30] J. Rättyä, Linear differential equations with solutions in function spaces, to appear in Complex Var. Elliptic Equ.

[31] S. A. Vinogradov, Multiplication and division in the space of analytic functions with an area-integrable derivative, and in some related spaces (Russian), Zap. Nauchn. Sem. S.-Peterburg. Otdel. Mat. Inst. Steklov (POMI), 222 (1995), Issled. po Linein. Oper. i Teor. Funktsii, no. 23, 45-77, 308; Translation in J. Math. Sci. (New York), 87 (1997), no. 5, 3806-3827.

[32] H. Wittich, Zur Theorie linearer Differentialgleichungen im Komplexen, Ann. Acad. Sci. Fenn. Ser. A. I., 379 (1966), 1-18.

[33] R. Zhao, On a general family of function spaces, Ann. Acad. Sci. Fenn. Diss., 105 (1996), 1-56.

[34] K. Zhu, Operator theory in function spaces, Marcel Dekker, New York, 1990.

[35] K. Zhu, Bloch type spaces of analytic functions, Rocky Mountain J. Math., 23 (1993), no. $3,1143-1177$. 
J. Heittokangas

University of Joensuu

Mathematics

P.O. Box 111

FI-80101 Joensuu

FINLAND

janne.heittokangas@joensuu.fi

R. Korhonen

University of Joensuu

Mathematics

P.O. Box 111

FI-80101 Joensuu

FINLAND

risto.korhonen@joensuu.fi

J. Rättyä

University of Joensuu

Mathematics

P.O. Box 111

FI-80101 Joensuu

FINLAND

jouni.rattya@joensuu.fi 